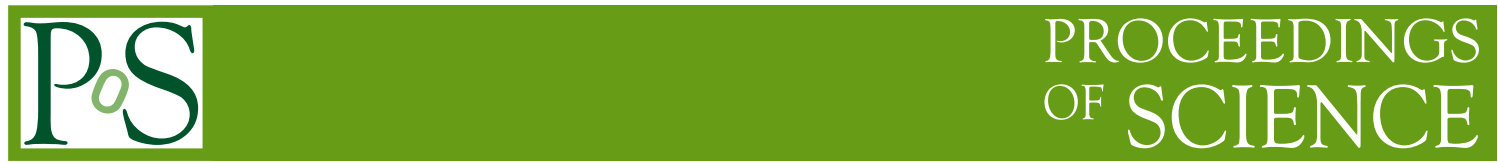

\title{
Discussion of input data and possible results for the ${ }^{12} \mathbf{C}(\alpha, \gamma){ }^{16} \mathrm{O}$ problem
}

\section{Lothar Buchmann*}

TRIUMF

E-mail: lotharatriumf.ca

10th Symposium on Nuclei in the Cosmos

July 27 - August 12008

Mackinac Island, Michigan, USA

\footnotetext{
${ }^{*}$ Speaker.
} 


\section{Introduction}

The ${ }^{12} \mathrm{C}(\alpha, \gamma){ }^{16} \mathrm{O}$ reaction remains an ongoing problem in Nuclear Astrophysics and has thus drawn lots of attention over the past decades. The reason is for one its exceptional importance for the nucleosynthesis products and even the dynamics of massive stars. The other reason is that it is extremely difficult to determine the reaction cross section at astrophysically relevant energies.

The reason for the latter is that the ${ }^{12} \mathrm{C}(\alpha, \gamma){ }^{16} \mathrm{O}$ reaction cross section is made up by contributions from several transitions with many wide resonances in the relevant regions. Measurements show that the ground state transition in ${ }^{16} \mathrm{O}$ is dominant at low energies with some contributions from cascades. The ground state radiative capture, however, has two electromagnetic components, an electric dipole and an electric quadrupole one, coherent in the $\gamma$-angular distribution, incoherent in the total cross section. All these cross sections need to be extrapolated to low energies, in particular to a center of mass energy of $300 \mathrm{keV}$ equivalent to static core helium burning.

To constrain the extrapolations one uses the following input:

- The basic nuclear structure of ${ }^{16} \mathrm{O}$ is considered to be sufficiently known. In particular, tabulated state values can be reasonably trusted and no new states are introduced.

- There are many direct measurements of the ${ }^{12} \mathrm{C}(\alpha, \gamma){ }^{16} \mathrm{O}$ reaction that must be taken into account. Normal procedure is to take them with equal weight.

- $\alpha$-widths can be frequently determined independent of the radiative capture data, e.g. by elastic scattering data or the $\beta$-delayed $\alpha$-decay of ${ }^{16} \mathrm{~N}$.

- $\gamma$ widths of subthreshold have been determined by electron scattering and are quoted with a small error. In addition, $\gamma$-decay branching ratios of the ${ }^{16} \mathrm{O}$ states in question have been frequently measured by populating these states by several kind of reactions.

- Transfer reactions, ANC methods, and Coulomb breakup, also allow, in principle, to determine $\alpha$-widths. However, all these methods are somewhat model dependent and errors are hard to estimate.

Typically R-matrix fits [1] are used to do the extrapolations to low energies. These allow to connect the radiative scattering data to the elastic scattering data and the $\beta$-delayed $\alpha$-decay spectrum of ${ }^{16} \mathrm{~N}$. E.g. the $E 1$ radiative capture data to the ground state are fitted with ${ }^{1}$

$$
\sigma_{E 1}^{0}(E)=\frac{6 \pi}{k^{2}} P_{1}(E)\left|\frac{\sum_{\lambda} \frac{\gamma_{\lambda} \Gamma_{\lambda \gamma}^{1 / 2}}{E_{\lambda}-E}}{1-\left(S_{1}(E)-B_{1}+i P_{1}(E)\right) R_{1}(E)}\right|^{2}
$$

and the energy dependent $\gamma$-width

$$
\Gamma_{\lambda \gamma}=2 E_{\gamma}^{3} \gamma_{\lambda \gamma}^{2}
$$

\footnotetext{
${ }^{1}$ For $R$-matrix notation, see Ref. [1, 2, 3].
} 
for the radiative capture and including

$$
W(E)=f_{\beta}(E) \sum_{\ell=1,3} P_{\ell}(E)\left|\frac{\sum_{\lambda} \frac{A_{\lambda \ell}}{E_{\lambda \ell}-E}}{1-\left[S_{\ell}(E)-B_{\ell}+i P_{\ell}(E)\right] R_{\ell}}\right|^{2}
$$

for the fit of the ${ }^{16} \mathrm{~N} \beta$-delayed $\alpha$-spectrum. Here the index $\lambda$ indicates summation over states, The index $1(\ell)$ indicating an angular moment of $1(\ell)$, the index $\gamma$ indicates that the symbol refers to radiative properties. $P(E)$ with an energy indicator refers to the $\ell=1$ penetrability, $S(E)$ are the shift functions and $B$ the boundary conditions. The $R$-function is given by

$$
R_{\ell}(E)=\sum_{\lambda} \frac{\gamma_{\lambda \ell}}{E_{\lambda \ell}-E}
$$

For the ground state $E 2$ transition in ${ }^{12} \mathrm{C}(\alpha, \gamma){ }^{16} \mathrm{O}$ is

$$
\sigma_{E 2}^{0}=\frac{5 \pi}{k^{2}}\left|U_{0}^{2}(E)\right|^{2}
$$

with the scattering function

$$
U_{0}^{2}(E)=-i e^{i \Omega_{2}} 2 P_{2}^{1 / 2} k_{\gamma}^{5 / 2}\left[\sum_{\lambda \mu} \gamma_{\lambda}^{2} \gamma_{\mu \gamma 0}^{2} A_{\lambda \mu}^{2}+\frac{3}{\sqrt{10}} \frac{M_{n}^{1 / 2} e}{\hbar k} N_{f}^{1 / 2} a^{2} F_{2}(a) G_{2}(a) \theta_{\alpha}^{0}(2200 \mid 00) J_{2}^{\prime}(2,0)(E)\right]
$$

where the $A_{\lambda \mu}$ are the elements of the state matrix[1], $\lambda \mu$ are state number indices. $\Omega_{2}=\omega_{2}-\phi_{2}$, $\omega_{2}$ is the Coulomb phase shift, $\phi_{2}$ the hard sphere phase shift [1], $P_{2}$ the $\ell=2$ penetrability, $k_{\gamma}=\frac{E_{\gamma}}{\hbar c}$ the radiative wave number, $M_{n}$ the atomic mass unit, $e_{q}$ the electric unit charge, and $F_{2}(a)$ and $G_{2}(a)$ the regular and irregular Coulomb functions at the radius $a$, respectively. Therein is $M_{n}$ the atomic mass unit $\left(931 \mathrm{MeV} / \mathrm{c}^{2}\right)$. The normalization of the wave functions is

$$
N_{f}^{-1}=1+\frac{2\left(\theta^{0}\right)^{2}}{a} \int_{a}^{\infty} d r\left[\frac{W_{0}(r)}{W_{0}(a)}\right]^{2}
$$

with the dimensionless reduced width amplitude for the $\alpha$-particle

$$
\theta_{\alpha}^{0}=\gamma^{0}\left(\hbar^{2} / \mu a\right)^{-1 / 2}
$$

$W_{J}(r)$ are the Whittaker functions, i.e. to a very good approximation the continuation of the bound state wavefunction outside the channel radius $a$. In similar fashion

$$
J_{2}^{\prime}(2,0)(E)=\frac{1}{a^{3}} \int_{a}^{\infty} d r r^{2} \frac{W_{0}(r)}{W_{0}(a)}\left[\frac{F_{2}(r)}{F_{2}(a)}-\frac{G_{2}(r)}{G_{2}(a)}\right] .
$$

Note the overlap between the Whittaker ground state function (index 0) and the Coulomb scatter functions (index 2) in the integral $J_{2}^{\prime}(2,0)$.

The $\gamma$-width $\gamma_{\mu \gamma 0}^{2}$ can in principle be divided into internal and external regions

$$
\gamma_{\mu \gamma 0}^{2}=\gamma_{\mu \gamma 0}^{2}(\text { int })+\gamma_{\mu \gamma 0}^{2}(\text { chan })
$$

with the internal part normally dominant and the channel part given by a similar expression to the direct one, i.e the right part of the sum in Eq. 1.6 [1].

The direct, non resonant part of the cross section is most important in the $E 2$ transitions both for the ground state as well as the excited states. 


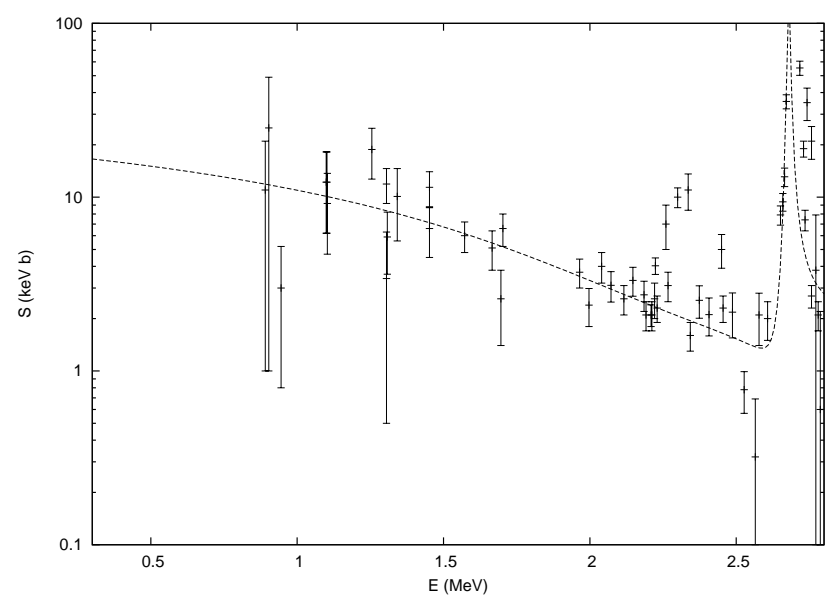

Figure 1: The combined Kunz and Fey $E 2$ ground state data and a fi t to them.

\section{Analysis of the Stuttgart data}

In this section we present a short look on the Stuttgart data as they are available from the Refs. $[4,5,6,7,8,9,10]$. As the Stuttgart group places strong emphasis on including the well known $2^{+}$ resonances at $\mathrm{E}=4.4$ and $\mathrm{E}=5.9 \mathrm{MeV}$, they have been included here as well. Angular distributions have been taken from the doctoral theses and are included in the fits.

For a test, if the non-inclusion of the direct capture has any consequence for the absolute value of $\mathrm{S}_{E 2}(300)$ as derived in in the above references all data sets quoted in the theses of R. Kunz [9] and M. Fey [10] (as this should give the best statistical constraint) have been combined and made subject to a fit. This includes higher lying resonance information and, of course, the DC expression of Ref. [1], Eq. 1.6. The fit shown in Fig. 1 is obtained. Obviously $S_{E 2}(300)(11 \mathrm{keV} \mathrm{b})$ is far outside the range of values allowed in Refs. $[4,5,6,7,9,10]$. As far as one can tell the least squares parameter $\chi^{2}$ is not considerably different from the fits shown in the article. As noticed, the total $\chi^{2}$ is largely produced by the data starting at about and above $2 \mathrm{MeV}$. In fact, the low energy data seem to be nearly irrelevant to the quality of the fit. As the authors of the above references also point out correctly, in the $2 \mathrm{MeV}$ energy region the $E 1$ cross sections are quite dominant and the $E 2$ data in this region can be subject to large systematic errors. In fact, the $E 1$ and $E 2$ data derived from angular distributions are always correlated, a point not considered in many previous error analyses, see more in Sec. 5.

\section{Phaseshift analysis}

Several measurements $[11,12,13,14,15,16,17,18,19,20,21,22,23,24]$ of the elastic ${ }^{12} \mathrm{C}(\alpha, \alpha){ }^{12} \mathrm{C}$ reaction have been made as early as 1953 . While there have been some elastic data taken with a carbon beam on a helium gas target in the context of radiative capture measurements, all of the explicit elastic scattering measurements employed $\alpha$ (helium) beams on thin carbon foils and measured scattered $\alpha$ angular distributions. The numbers of angles measured varies from two to 32 ; different angles were determined sometimes by rotating the detector table, or by providing a large number of fixed detectors. A typical experiment is described in Ref. [24]. Normally the 
scattering data have then undergone phase shift analysis and only the derived phase shifts have been published, sometimes without error bars. Only a few measurements have provided phase shift error bars [19, 22], and only one [22] has made the primary data available. While phaseshift analyses are preferred in theoretical modeling (like potential models) as they separate states and partial waves of different spins, there are inherent problems connected with the non-linearity of the analysis [2].

Ref. [24] describes an elastic ${ }^{12} \mathrm{C}+\alpha$ scattering experiment in which a global $R$-matrix analysis has been employed. In the analysis, the reduced width amplitude $\gamma_{12}$ of the $2^{+}$subthreshold state $\left(\mathrm{E}_{x}=6.917 \mathrm{MeV}^{2}, \mathrm{E}=-245 \mathrm{keV}^{3}\right)$. was varied. $\mathrm{S}_{E 2}(300)$ of the radiative ground state transition scales approximately with the square of the reduced width amplitude of the $2^{+}$sub-threshold state. The minimum in the least squares distribution occurred for $\gamma_{12}=0.47 \mathrm{MeV}^{1 / 2}$, and for the $1^{-}, 7.1$ $\mathrm{MeV}$ reduced width amplitude (that was also varied) at $\gamma_{11}=0.27 \mathrm{MeV}^{1 / 2}(a=5.5 \mathrm{fm}$ ). To obtain an error estimate, fits were obtained for values of $\gamma_{12}$ from 0.2 to $0.60 \mathrm{MeV}^{1 / 2}$, with all other parameters allowed to vary. The same approach was used to scan $\gamma_{11}$ from 0 to $0.60 \mathrm{MeV}^{1 / 2}$ for the $1^{-}$state. $1 \sigma$ uncertainties of $\gamma_{12}=0.47 \pm 0.06 \mathrm{MeV}^{1 / 2}$, and $\gamma_{11}=0.27_{-0.27}^{+0.11} \mathrm{MeV}^{1 / 2}$ were calculated with the previously established [3] guideline $\chi^{2}<\chi_{\min }^{2} \pm 9 \chi_{v}^{2}$. This has to be compared to $\gamma_{11}=0.18 \pm 0.02 \mathrm{MeV}^{1 / 2}$ of Ref. [3]. The best fit has a $\chi_{v}^{2}$ of approximately 1.66. Deviations from an ideal fit occurred at resonances with widths in the $\mathrm{keV}$ range where the sensitivity to target effects and beam energy calibration would be most pronounced.

A detailed description of the Notre Dame experiment including a Monte Carlo based phaseshift derivation is about to be published [25].

\section{The $\beta$-delayed $\alpha$-decay of ${ }^{16} \mathbf{N}$}

Recently two articles have been published [26, 28] describing measurements of the $\beta$-delayed $\alpha$ decay of ${ }^{16} \mathrm{~N}$. They also contain some statements about the $\beta$-delayed $\alpha$-spectrum of ${ }^{16} \mathrm{~N}$ measured at TRIUMF [3]. We have revisited all measurements and have made GEANT4 calculations of these experiments. The results will be published in Ref. [29].

From our calculations we come to these general conclusions:

- All measured ${ }^{16} \mathrm{~N}$ spectra are highly sensitive to target and detector responses.

- These responses can be partially suppressed, e.g. by coincidence methods.

- However, once part of the spectrum they can not be corrected for.

- The corrections applied to the Yale spectra $[27,26]$ are mathematically incorrect. The final spectra derived are therefore meaningless.

- The Mainz spectrum [30, 31] is not a 'zero target thickness' [27] spectrum and has no suppression of responses. Indeed it is visibly broadened by response.

- Simulations show that most spectra measured are consistent with the final TRIUMF spectrum after background subtraction and energy calibration.

\footnotetext{
${ }^{2}$ For conciseness in the subsequent text, state energies will be truncated to two signifi cant fi gures and may be used as indices for symbols, e.g. $\theta_{\alpha}^{6.9}$.

${ }^{3}$ ' $E$ ' labels the center-of-mass energy in any system discussed in this article.
} 

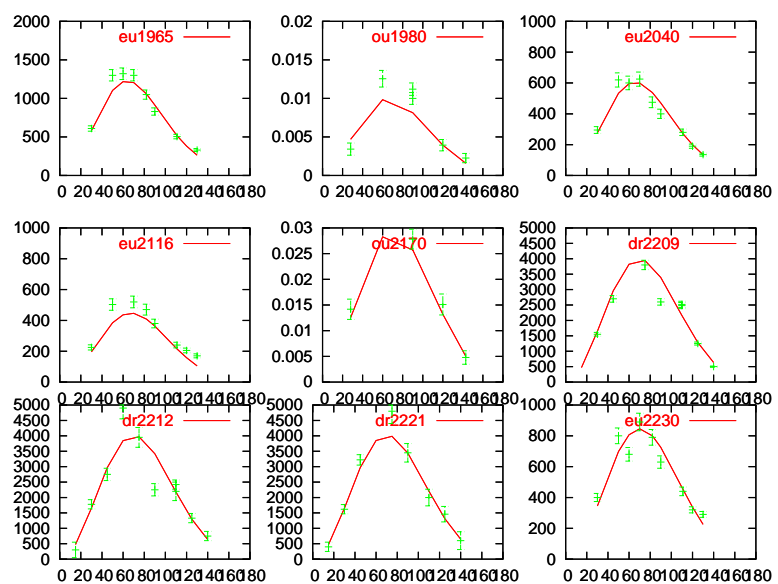

Figure 2: Some fi ts of $\gamma$-angular distribution data. Indicated are the energies and the origin of the spectra (eu: Stuttgart Eurogam, Ou: Ouellet et al. [32], dr: Stuttgart Drehtisch.

- The Argonne spectrum [28] may suffer from background problems.

- The background subtraction used to derive the TRIUMF spectrum does not account for differences between spectra.

\section{A global fit of most data}

A global fit to most of the available data has been performed using in particular the phaseshifts of Ref. [25] as well as the Stuttgart angular $\gamma$-distributions extracted from the theses work. Some fits to $\gamma$-angular distributions of different measurement are shown in Fig. 2 Most fits are of reasonable quality, but not perfect, as can be expected for a global fit.

The results can be summarized in the subsequent plots: While stepping the reduced width amplitude $\gamma_{12}$ of the $2^{+}$subthreshold state one obtains the least squares dependence shown in Fig. 3. Obviously, high values of $\gamma_{12}$ can be excluded, corresponding approximately to $\mathrm{S}_{E 2}(300)$ of more than $60 \mathrm{keV} \mathrm{b}$. There are apparently two minima, corresponding to a destructive and a constructive solution as shown in Fig. 4. It may be noted that both minima occur at approximately the same absolute amplitude of $\gamma_{12}$. This is caused by the inclusion of the elastic scattering phase shift of Ref. [25] that forces the reduced width amplitude of the $6.9 \mathrm{MeV}$ subthreshold state to values around the minima, see Sec. 3.

The dependence of the reduced width amplitude $\gamma_{12}$ and the dimensionless reduced width of the ground state $\left(\theta_{10}\right)$ is shown in Fig. 5. From Fig. 5 it is clear that the destructive interference is associated with an absolute dimensionless reduced width of the ground state of close to one. From nuclear structure considerations such a case can be pretty well excluded. Choosing the constructive interference minimum results in a value of $S_{E 2}(300)=45 \mathrm{keV}$ b with $60 \mathrm{keV}$ b as an upper limit. A lower limit is harder to determine and the maximum between the two minima is chosen here corresponding to $\mathrm{S}_{E 2}(300)=10 \mathrm{keV} \mathrm{b}$, thus resulting in a highly asymmetric error.

In general, the Stuttgart data fitted by themselves result in a low $S_{E 2}(300)$ (see Sec. 2). This forces a lowering of $S_{E 1}(300)$, as the angular distribution data fix the ratio between the two cross 

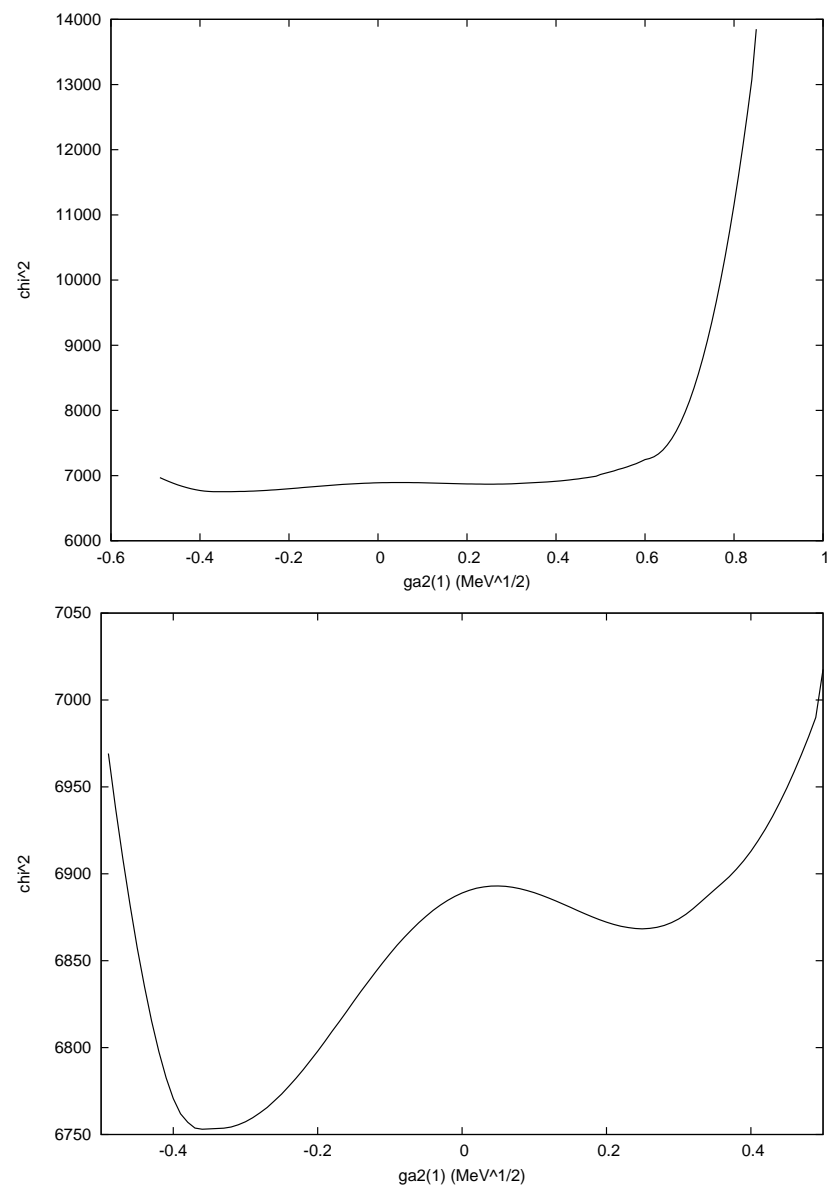

Figure 3: The least squares dependence of the parameter $\gamma_{12}$ for the fi t described in the text. The upper panel shows the full fi tting range, the lower one the region of the two minima.

sections, while the reduced $\alpha$-width of the subthreshold $1^{-}$state is held close to constant by the $\beta$-delayed $\alpha$-spectrum of ${ }^{16} \mathrm{~N}$. Thus $\mathrm{S}_{E 1}(300)=70 \mathrm{keV}$ b is found for which we attach an error of $20 \mathrm{keV} \mathrm{b}$ as previously done ([3]).

As for cascade transitions, for the transition into the first excited $0^{+}$state of ${ }^{16} \mathrm{O}$, we assign 25 $\mathrm{keV} \mathrm{b}$, with an error of $16 \mathrm{keV} \mathrm{b}$ (Ref. [34]). Without further discussion, for the cascade transition into the $6.9 \mathrm{MeV}^{+}$state a value of $S_{6.9}(300)=10 \pm 6 \mathrm{keV} \mathrm{b}$ is chosen.

Added up, the total $\mathrm{S}$-factor at a center of mass energy of $300 \mathrm{keV}$ in ${ }^{12} \mathrm{C}(\alpha, \gamma){ }^{16} \mathrm{O}$ can be estimated to

$$
S(300)=150_{-77}^{+57} \mathrm{keVb}
$$

which is not a major change compared to the value of Ref. [2]. 

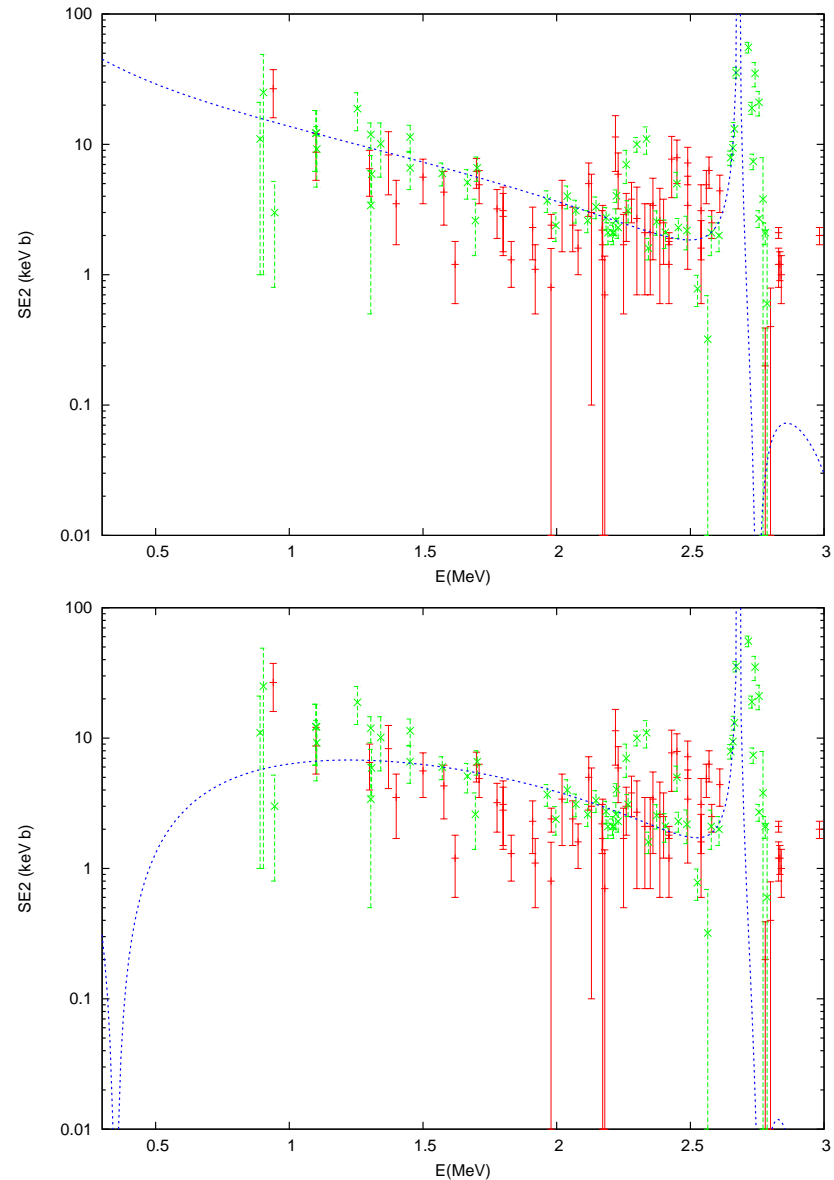

Figure 4: Two fi ts to the combined data ( $E 2$ shown, red: Stuttgart data $[4,9,6,7,8,9,10]$, green: other radiative capture data, for a full reference list see [33].) with a constructive (upper panel) and destructive solution (lower panel) corresponding to the minima shown in Fig. 3.

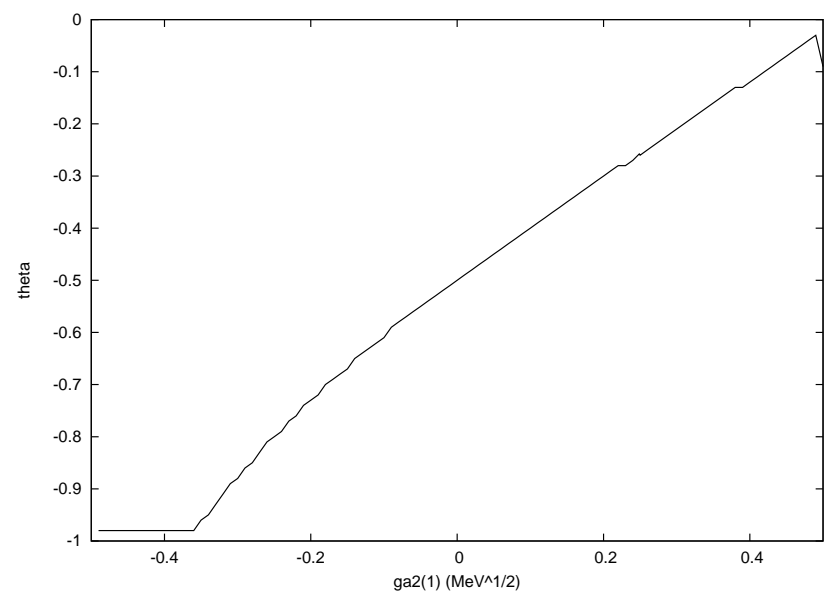

Figure 5: Dependence of the dimensionless reduced width of the ground state from the reduced width amplitude of the subthreshold state. 


\section{References}

[1] F.C. Barker and T. Kajino, Aust. J. Phys., 44369 (1991).

[2] L. Buchmann, R.E. Azuma, C.A. Barnes, J. Humblet, K. Langanke, Phys. Rev. C, 54, 354 (1996).

[3] R.E. Azuma, L. Buchmann, F.C. Barker, C.A. Barnes, J.M. D’Auria, M. Dombsky, U. Giesen, K.P. Jackson, J.D. King, R.G. Korteling, P. McNeely, J. Powell, G. Roy, J. Vincent, T.R. Wang, S.S.M. Wong, and P.R. Wrean, Phys. Rev. C, 50, 1194 (1994); an initial report was given in L.Buchmann, R.E.Azuma, C.A.Barnes, J.D' Auria, M.Dombsky, U.Giesen, K.P. Jackson, J.D.King, R.Korteling, P.McNeely, J.Powell, G.Roy, J.Vincent, T.R.Wang, S.S.M.Wong, and P.W.Wrean, Phys. Rev. Lett. 70 726 (1993).

[4] R. Kunz, M. Jaeger, A. Mayer, J.W. Hammer, G. Staudt, S. Harissopulos, and T. Paradellis, Phys. Rev. Lett., 863244 (2001).

[5] R. Kunz, M. Fey, M. Jaeger, A. Mayer, J.W. Hammer, G. Staudt, S. Harissopulos, and T. Paradellis, Astr. Phys. J. 567643 (2002).

[6] J.W. Hammer, M. Fey, R. Kunz, J. Kiener, V. Tatischeff, F. Haas, M. Assunção, C. Beck, C. Boukari-Pelissie, A. Coc, J.J. Correia, S. Courtin, F. Fleurot, E. Galanopoulos, C. Grama, F. Hammache, S. Harissopulos, A. Korichi, E. Krmpotiĉ, D. Le Du, A. Lopez-Martens, D. Malcherek, R. Meunier, P. Papka, T. Paradellis, M. Rousseau, N. Rowley, G. Staudt, S. Szilner Nucl. Phys. A752 $514 c(2005)$.

[7] J.W. Hammer, M. Fey, R. Kunz, J. Kiener, V. Tatischeff, F. Haas, M. Assunção, C. Beck, C. Boukari-Pelissie, A. Coc, J.J. Correia, S. Courtin, F. Fleurot, E. Galanopoulos, C. Grama, F. Hammache, S. Harissopulos, A. Korichi, E. Krmpotiĉ, D. Le Du, A. Lopez-Martens, D. Malcherek, R. Meunier, P. Papka, T. Paradellis, M. Rousseau, N. Rowley, G. Staudt, S. Szilner Nucl. Phys. 758 363c (2005).

[8] M. Assunção, M. Fey, A. Lefebvre-Schuhl, J. Kiener, V. Tatischeff, J.W. Hammer, C. Beck, C. Boukari-Pelissie, A. Coc, J.J. Correia, S. Courtin, F. Fleurot, E. Glanopoulos, C. Grama, F. Hass, F. Hammache, F. Hannachi, S. Harissopoulos, A. Korichi, R. Kunz, D. LeDuc, A. Lopez-Marteros, D. Malcherek, R. Meunier, Th. Paradellis, M. Rousseau, N. Rowley, G. Staudt, S. Szilner, J. P. Thibaud, and J.L Weil, Phys. Rev. C 73, 055801 (2005).

[9] R. Kunz, Doctoral thesis, http://elib.uni-stuttgart.de/opus/volltexte/2003/1266, Stuttgart (2003).

[10] M. Fey, Doctoral thesis, http://elib.uni-stuttgart.de/opus/volltexte/2004/1683, Stuttgart (2004).

[11] R.W. Hill, Phys. Rev., 90845 (1953).

[12] J.W. Bittner and R.D. Moffat, Phys. Rev., 96374 (1954).

[13] C.M. Jones, G.C. Phillips, R.W. Harris and E.H. Beckner, Nucl. Phys., 371 (1962).

[14] J.D. Larson and T.A. Tombrello, Phys. Rev., 147760 (1966).

[15] G.J. Clark, D.J. Sullivan and P.B. Treacy, Nucl. Phys. A, 110481 (1968).

[16] J.M. Morris, G.W. Kerr and T.R. Ophel, Nucl. Phys. A, 11297 (1968).

[17] T.P. Marvin and P.P. Singh, Nucl. Phys. A, 180282 (1972).

[18] F. Brochard, P. Chevallier, D. Disdier, V. Rauch and F. Scheibling, Journal de Physique, 36113 (1975).

[19] M. D’ Agostino Bruno, I. Massa, A. Uguzzoni, G. Vannini, Nuovo Cimento A, 27, 1 (1975). 
[20] A.D. Frawley, J.D. Fox, K.W. Kemper and L.C. Dennis, Phys. Rev. C, 252935 (1982).

[21] M.A. Kovash, R.W. Lourie, W. Pugh, C.E. Hyde-Wright, D.G. Marchlenski, H.R. Suiter, J.C. Brown and R.G. Seyler, Phys. Rev. C, 311065 (1985).

[22] R. Plaga, H.W. Becker, A. Redder, C. Rolfs, H.P. Trautvetter, Nucl. Phys. A, 465, 291 (1987).

[23] S.Y. Tong, W.N. Lennard, P.F.A. Alkemade and I.V. Mitchell, Nucl. Instr. and Methods B45 30 (1990).

[24] P. Tischhauser, R.E. Azuma, L. Buchmann, R. Detwiller, U. Giesen, J. Görres, M. Heil, J. Hinnefeld, F. Käppler, J.J. Kolata, H. Schatz, A. Shotter, E. Stech, S. Vouzoukas, and M. Wiescher, Phys. Rev. Lett., 88072501 (2002).

[25] P. Tischhauser et al., to be published (2008).

[26] R.H. France III, E.L. Wilds, N.B. Jevtic, J.E. McDonald, and M. Gai, Nucl. Phys. A621, $165 c, 1997$.

[27] Z.Zhao, R.H. France III, K.S. Lai, S.L. Rugari, M. Gai, and E.L. Wilds, Phys. Rev. Lett. 70, 2066 (1993).

[28] X.D. Tang, K.E. Rehm, I Ahmad, C. Brune, A. Champagne, J. Greene, A.A. Hecht, D. Henderson, R.V.F. Janssens, C.R.L. Jiang, L. Jisonna, D. Kahl, E.F. Moore, M. Notani, R.C. Pardo, N. Patel, M. Paul, G. Savard, J. P. Schiffer, R. E. Segel, S. Sinha, B. Shumard, and A. Wuosma, Phys. Rev. Let. 99, 052502 (2007).

[29] L. Buchmann, G. Ruprecht, and C. Ruiz, Physical Review C, in press (2008).

[30] K.Neubeck, H. Schober and H. Wäffler Phys. Rev. C 10, 320 (1974).

[31] H. Wäffler, private communication to C.A. Barnes, Dec. 16, 1997.

[32] J.M.L. Ouellet, N. Butler, H.C. Evans, H.W. Lee, J.R. Leslie, J.D. MacArthur, W. McLatchie, H.-B. Mak, P. Skensved, J.L. Whitton, X. Zhao, Phys. Rev. C, 4, 1982 (1996).

[33] L. Buchmann and C.A. Barnes, Nucl. Phys. A, 777c (2006) 254.

[34] C. Matei, L. Buchmann, WR. Hannes, D.A. Hutcheon, C. Ruiz, C.R. Brune, J. Caggiano, A.A. Chen, J. D’Auria, A. Laird, M. Lamey, ZH. Li, WP. Liu, D. Ottewell, J. Pearson, G. Ruprecht, M. Trinczek, C. Vockenhuber, and C. Wrede, Phys. Rev. Lett. 97242503 (2006) 